\title{
A Magnetically Shielded Instrument for Magnetoresistance and Noise Characterizations of Magnetic Tunnel Junction Sensors
}

\author{
Z. Q. Lei, G. J. Li, P. T. Lai, Philip W. T. Pong \\ Department of Electrical and Electronic Engineering \\ The University of Hong Kong \\ Pokfulam Road, Hong Kong \\ ppong@eee.hku.hk
}

\author{
William. F. Egelhoff, Jr. \\ Magnetic Materials Group \\ National Institute of Standards and Technology \\ Gaithersburg, MD 20899-8552, USA
}

\begin{abstract}
A magnetically shielded setup was developed for characterizing magnetoresistance (MR) and noise properties of magnetic tunneling junction (MTJ) sensors. A mu-metal shielding is installed to avoid the interference of external magnetic disturbance. Both MR curves and noise power spectra of MTJ sensors can be obtained for further data analysis. Moreover, a hard-axis magnetic field can be applied to eliminate the hysteresis and the linear field response of MTJ sensors can be measured. The preliminary measurement results on MTJ sensors are presented to illustrate the characterization capabilities of this setup.
\end{abstract}

Keywords-1/f noise; mangetic tunnel junction (MTJ); tunneling magnetoresistance (TMR); measurement setup

\section{INTRODUCTION}

Magnetic field sensors have been used for a long time due to their extensive applications ranging from compass for navigation to harddisk drives for data storage. With the continuous development of low-field detections and highdensity storage applications, there is a growing demand of magnetic sensors with detectivity down to the scale of picoTesla [1]. However, nowadays the detection of magnetic fields in the regime between 1 nanoTesla and 1 picoTesla is dominated by relatively large, expensive, power-consuming magnetometers such as fluxgates, search-coil, optically pumped magnetometry, and superconducting quantum interference devices (SQUIDs) [2]. Therefore, an alternative low-field magnetic sensor which possesses small size, low cost, and low-power properties will bring about important technological impacts.

Magnetic tunneling junctions (MTJs) are regarded as a competitive candidate for ultra-low magnetic field detection due to their low cost, high sensitivity and large tunneling magnetoresistance (TMR) ratio. Fig. 1 illustrates the schematic structure of a MTJ which consists of bottom electrode, ferromagnetic (FM) free layer, tunneling barrier, ferromagnetic (FM) pinned layer, anti-ferromagnetic pinning layer, and top electrode. The TMR is defined by the Julliere's spin-polarized tunneling model [3], TMR $=2 \mathrm{P}_{1} \mathrm{P}_{2} /\left(1-\mathrm{P}_{1} \mathrm{P}_{2}\right)=\left(\mathrm{R}_{\mathrm{AP}}-\mathrm{R}_{\mathrm{P}}\right) / \mathrm{R}_{\mathrm{P}}$, where $\mathrm{P}_{1}$ and $\mathrm{P}_{2}$ are the spin polarizations of the two $\mathrm{FM}$ free layers at the Fermi surface, $R_{P}$ and $R_{A P}$ are the resistances when the two

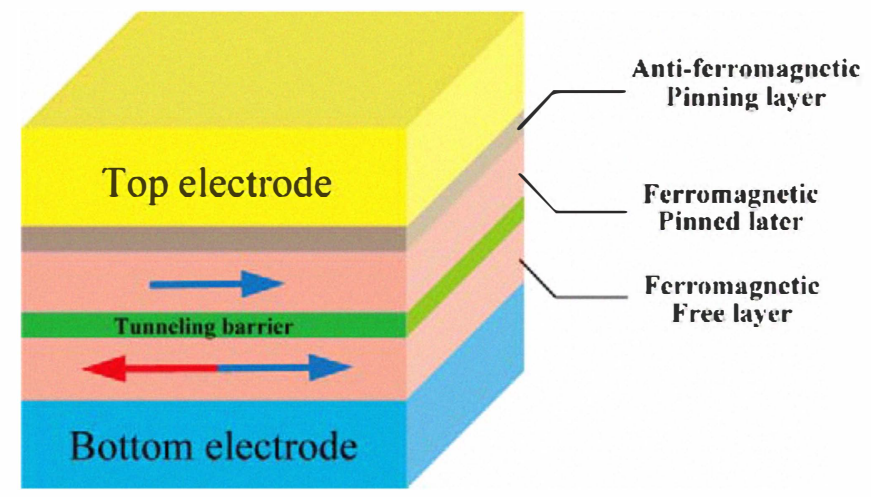

Figure 1. Schematic drawing of a MTJ. The arrows indicate the possible magnetization directions.

FM layers are aligned in parallel and anti-parallel configurations respectively. The fundamental working principle of a MTJ is the variation of junction resistance with the relative orientation between the two ferromagnetic layers. The magnetization direction of a free layer can be rotated by an external magnetic field. The MTJ resistance is larger when the magnetizations of the two FM layers (free layer and pinned layer) are opposite. On the contrary, the MTJ resistance is smaller when the magnetization directions are in the parallel state. MTJs with $1000 \%$ TMR [4] were theoretically predicted and $604 \%$ TMR [5] were experimentally demonstrated at room temperature. Therefore, MTJs are promising for applications in magnetic sensor industry. MTJ sensors can be applied in various areas such as biochips and biosensors [6-8], scanning MR microscopy [9], magnetocardiography and magnetoencephalography $[10,11]$, harddisk drive (HDD) reading heads [12] and magnetoresistive random access memory (MRAM) [13, 14]. MTJ sensors with a detectivity of 2 $\mathrm{pT} / \mathrm{Hz}^{1 / 2}$ at $500 \mathrm{kHz}$ was experimentally achieved by Chave et al. [15]. However, in many actual applications, the sensors have to operate in low-frequency regime (below $100 \mathrm{~Hz}$ ) where the signal-to-noise ratio (SNR) is greatly deteriorated by the intrinsic noises in MTJs. 


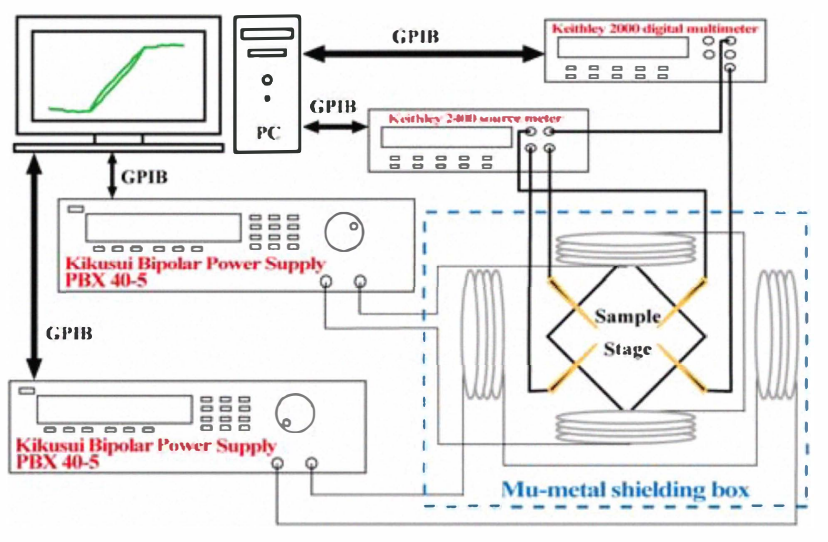

Figure 2. Schematic diagram of the instrument setup for TMR measurement. The MTJ is mounted on the sample stage and measured by four-probe electrical method. The sample stage and Helmholtz coils are shielded in the mu-metal magnetic shielding box. The sourcemeter provides a fixed current or fixed voltage across a MTJ and the digital multimeter measures the junction resistance. The Kikusui power supplies provide electric currents to the coils for generating magnetic fields. A sweeping magnetic field can switch a MTJ from parallel state to antiparallel state and vice versa. The TMR of the MTJ can then be determined from the variation of the junction resistance. The measurement data is conveyed to the PC via GPIB connection for further analysis.

The TMR and noise measurements are critical for characterizing MTJ sensors. The main noise sources in MTJs are amplifier noise, thermal noise, shot noise and $1 / f$ noise. The amplifier noise is not generated by MTJ junction itself, but from the amplifier in the external circuit. The thermal noise and shot noise are white noises and they are originated from the current fluctuations [16]. The $1 / f$ noise is attributed to charge trapping of electrons in barriers and at the interfaces of tunnel junctions and it is dominated in the low-frequency regime [1618]. We developed a magnetically shielded instrument setup in order to carry out TMR and noise characterizations.

\section{INSTRUMENTATION SETUP}

\section{A. TMR measurement system}

TMR measurement was performed by a four-point dc measurement method. The measuring setup is shown in Fig. 2. It is composed of a PC computer installed with LabVIEW software and PCI-GPIB interface, Keithley 2400 sourcemeter, Keithley 2000 digital multimeter, two sets of Kikusui Bipolar Power Supply PBX 40-5 and two pairs of Helmholtz coils. The Helmholtz coils and sample stage are shielded in a magnetic shielding box made of mu-metal. The shielding box can effectively divert the external magnetic field to go along the shielding instead of interrupting the sensors inside so as to reduce the disturbance from external magnetic field. The system is controlled by a program written in LabVIEW and the control computer communicates with the electrical instruments via GPIB cables. The Keithley 2400 sourcemeter serves to provide biasing current or voltage for MTJs and the Keithley 2000 digital multimeter was used to measure the junction resistance. The two sets of Kikusui Bipolar Power Supply PBX 40-5 are used to supply electric current to the coils so as to provide magnetic fields ranging from -200 Oe to $+200 \mathrm{Oe}$

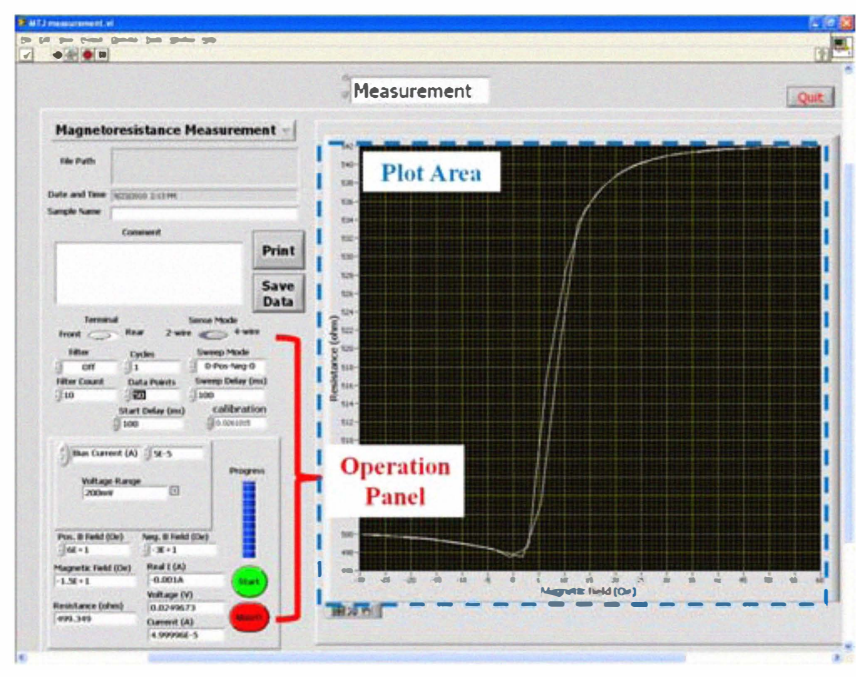

Figure 3. The interface of LabVIEW program for TMR measurement. On the left is the operation panel and on the right is the plot area.

along the easy-axis and hard-axis. The magnetic field in the easy-axis direction provides the sweeping field for MR-curve measurement while the hard-axis field is utilized to eliminate the hysteresis and enhance the linear response of MTJ sensors [19]. The whole system can be controlled at the interface of the LabVIEW control program as illustrated in Fig. 3.

\section{B. Noise measurement system}

Noise measurement circuit is shown in Fig. 4. It is composed of a Wheatstone bridge configuration, Keithley 2400 sourcemeter, Keithley 2000 digital multimeter, HP 34401A digital multimeter, Femto DLPVA-100-BLN-S low-frequency voltage amplifier, SR785 dynamic signal analyzer, two sets of Kikusui Bipolar Power Supply PBX 40-5 and the two pairs of Helmholtz coils. The bridge circuit is effective for eliminating the influence of thermal drift and dc offset [2]. The amplifier is required for amplifying the signal to a detectable level for data acquisition and spectral measurement. However, the amplifier itself is also a noise source and thus a low-noise instrumentation voltage amplifier (Femto DLPVA-100-BLN-S) is used in the setup. The bridge circuit, Helmholtz coils and amplifier are shielded in the magnetic shielding box. The noise measurement is conducted in the following procedure. First, a MTJ is mounted in one leg of the Wheatstone bridge circuit which is biased by the sourcemeter (Keithley 2400) in a constant current mode. The variable resistors in the other legs of the bridge circuit are adjusted until the electric potential over the two terminals (points A and B in Fig. 4) is equal which is measured by the digital multimeter (Keithley 2000). The junction voltage is detected by digital multimeter (HP 34401A). The output signal from the two terminals (points $A$ and $B$ ) is amplified through the amplifier and input to the dual-channel spectrum analyzer (Stanford Research Systems SR785). The spectrum analyzer carries out measurement in cross-correlation mode in order to effectively reduce the background noise floor of the measurement system. The biasing magnetic fields are provided by the two-axis Helmholtz coils. The sourcemeter (Keithley 2400) and the spectrum analyzer (Stanford Research 


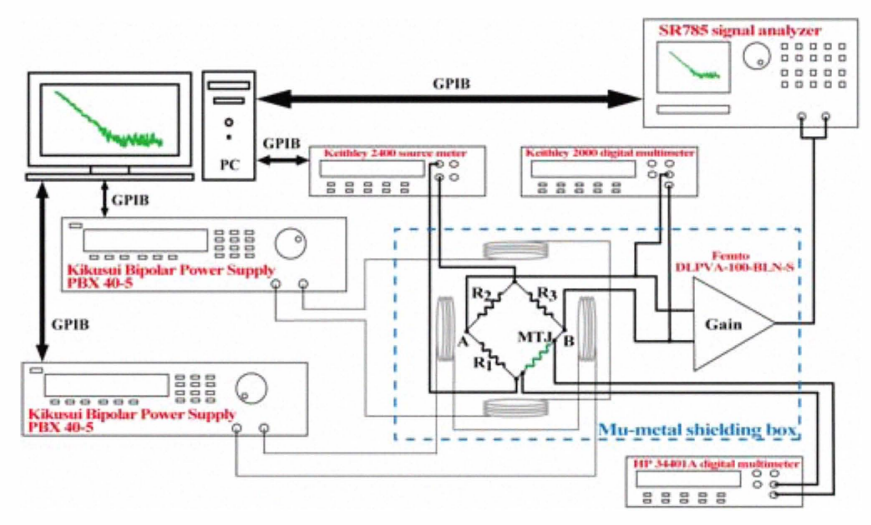

Figure 4. Schematic diagram of the instrument setup for MTJ noise characterization. The measurement is performed in Wheatstone bridge configuration. The MTJ is mounted in one leg of the bridge. $R_{1}, R_{2}$ and $R_{3}$ are variable resistors for balancing the output signal to zero. The output signal amplitude is amplified by the amplifier and input to the signal analyzer. The Helmholtz coils, bridge circuit and the amplifier are shielded in the mu-metal magnetic shielding box. The noise spectrum can be acquired by the PC through GPIB control.

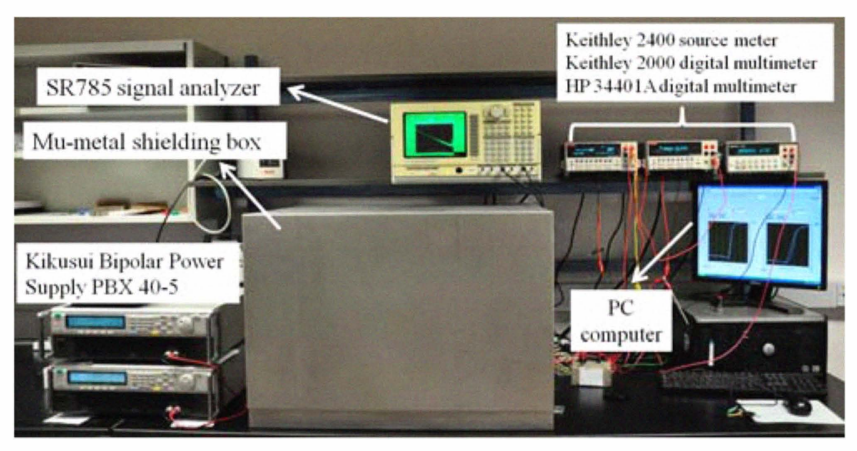

Figure 5. Facilities of magnetically shielded instrument for MR and noise characterizations of MTJ sensors.

Systems SR785) are connected to the PC computer via GPIB cables and it also can be controlled at the interface of the LabVIEW control program. The facilities of the measuring system for both MR and noise measurements are shown in Fig. 5 .

\section{PRELIMINARY MEASUREMENT RESUltS}

\section{A. TMR curves}

The MTJ samples were prepared by dc magnetron sputtering on thermally oxidized silicon wafers in an ultra-high vacuum chamber with a base pressure of $2 \times 10^{-10}$ Torr. The thin-film stack structure was substrate/ $\mathrm{Ni}_{77} \mathrm{Fe}_{14} \mathrm{Cu}_{5} \mathrm{Mo}_{4}$ 200/ $\mathrm{Co}_{50} \mathrm{Fe}_{50} 10 / \mathrm{Al} 10$ (oxidized)/ $\mathrm{Co}_{50} \mathrm{Fe}_{50} 10 / \mathrm{Ni}_{77} \mathrm{Fe}_{14} \mathrm{Cu}_{5} \mathrm{Mo}_{4}$ $25 / \mathrm{Co}_{50} \mathrm{Fe}_{50} 5 / \mathrm{Ir}_{20} \mathrm{Mn}_{80} 100 / \mathrm{Ru} 70$ (units in angstrom). The $\mathrm{Al}_{2} \mathrm{O}_{3}$ was formed by oxidizing the $\mathrm{Al}$ metal in the oxygen plasma. After depositing the thin films, the samples were annealed at $200{ }^{\circ} \mathrm{C}$ for $15 \mathrm{~min}$. Conventional self-aligned UV photolithography and ion etching process were followed to obtain the MTJs with the junction area of $20 \times 20 \mu \mathrm{m}^{2}$. The fabrication details are provided in [20]. Fig. 6 shows the

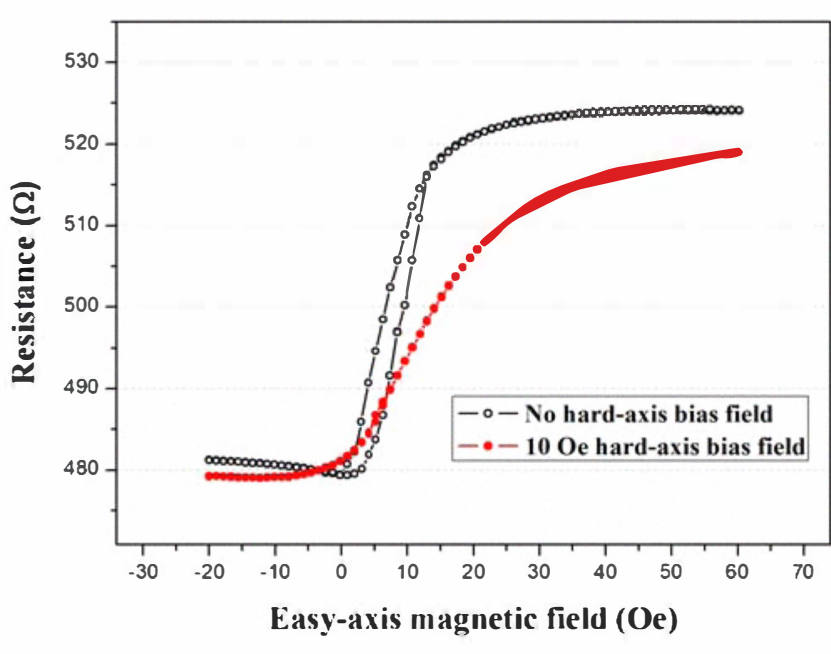

Figure 6. Resistance vs magnetic field measurement of the MTJ sample. TMR of $9.4 \%$ was measured without the hard-axis field. The hysteresis was then removed by applying a hard-axis field of 10 Oe. The TMR reduced to $8.3 \%$.

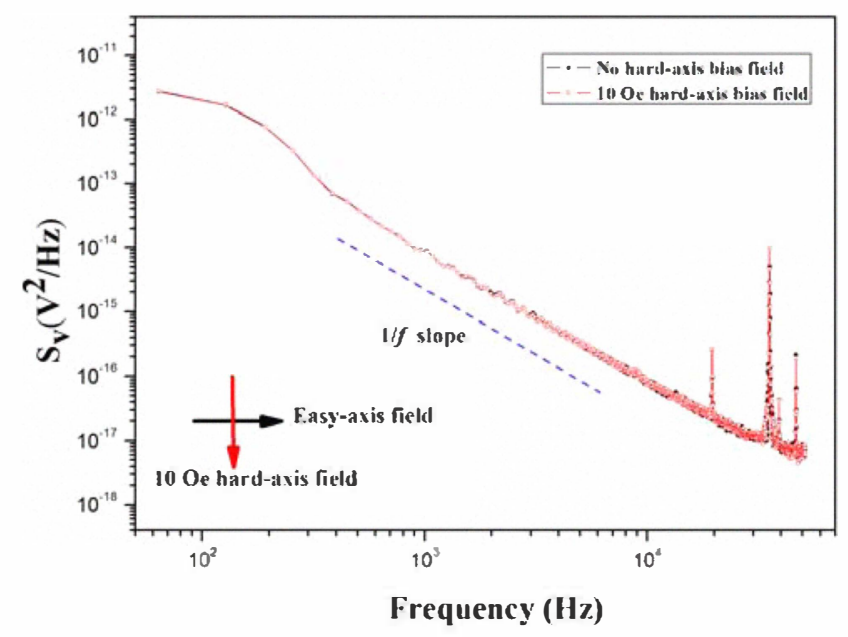

Figure 7. Noise spectrum of the MTJ sample. The voltage spectra of zero hard-axis and 10 Oe hard-axis bias nearly overlap with each other. The dashed line indicates $1 / f$ slope in the low-frequency regime.

resistance versus magnetic field measurement of the MTJ sample. The TMR and the coercivity were measured to be $9.4 \%$ and 5 Oe respectively. After applying a hard-axis field of $10 \mathrm{Oe}$, the TMR reduced to $8.3 \%$ with coercivity nearly eliminated. This removal of hysteresis is essential for MTJ sensors because a linear field response is needed for practical sensing applications $[19,21,22]$.

\section{B. Noise voltage spectra}

Fig. 7 shows the noise measurement results. The thermal noise and shot noise are white noise and they set a noise floor in the spectrum. The $1 / f$ noise is frequency-dependent and it is dominating in the low-frequency regime. The $1 / f$ noise is characterized by the Hooge parameter, $\alpha=A f S / V^{2}$, where $A$ is the junction area, $f$ is the frequency, $S$ is the voltage power of $1 / f$ noise and $V$ is the voltage across the junction. The Hooge 
parameter was calculated to be $5.5 \times 10^{-7} \mu^{2}$ and it is comparable with some previous works $[16,23]$. After applying a hard-axis field of $10 \mathrm{Oe}$, the Hooge parameter reduced to $5.3 \times 10^{-7} \mu \mathrm{m}^{2}$ and the decrease is attributed to the increase of junction voltage due to the hard-axis bias field.

\section{CONCLUSION}

We established a magnetically shielded setup for characterizing MR and noise properties of MTJ sensors. This setup is controlled by a computer through the LabVIEW interface. Both TMR curves and noise power spectra of MTJ sensors can be obtained with this instrument. A hard-axis magnetic field can also be applied to eliminate the hysteresis for linear response of the sensor. The preliminary measurement results show that this instrument is reliable and effective for characterizing the MTJ sensors.

\section{ACKNOWLEDGMENT}

This work was supported by the Seed Funding Program for Basic Research from the University of Hong Kong.

\section{REFERENCES}

[1] J. Lenz and S. Edelstein, "Magnetic sensors and their applications," Sensors Journal, IEEE, vol. 6, pp. 631-649, 2006.

[2] A. Edelstein, "Advances in magnetometry," Journal of Physics: Condensed Matter, vol. 19, pp. 165217-165244, 2007.

[3] M. Julliere, "Tunneling between ferromagnetic films," Physics Letters A, vol. 54, pp. 225-226, 1975.

[4] J. Mathon and A. Umerski, "Theory of tunneling magnetoresistance of an epitaxial Fe/MgO/Fe(001) junction," Physical Review B, vol. 63, p. 220403, 2001.

[5] S. Ikeda, et al., "Tunnel magnetoresistance of $604 \%$ at $300 \mathrm{~K}$ by suppression of $\mathrm{Ta}$ diffusion in $\mathrm{CoFeB} / \mathrm{MgO} / \mathrm{CoFeB}$ pseudo-spin-valves annealed at high temperature," Applied Physics Letters, vol. 93, p. 082508, 2008.

[6] W. Shen, X. Liu, D. Mazumdar and G. Xiao, "In situ detection of single micron-sized magnetic beads using magnetic tunnel junction sensors," Applied Physics Letters, vol. 86, pp. 253901-253903, 2005.

[7] F. A. Cardoso, et al., "Diode/magnetic tunnel junction cell for fully scalable matrix-based biochip," Journal of Applied Physics, vol. 99, pp. 08B307-303, 2006.
[8] P. P. Freitas and et al., "Magnetoresistive sensors," Journal of Physics: Condensed Matter, vol. 19, p. 165221, 2007.

[9] C. Mei-Lin, G. Jaramillo, S. Ahjeong, K. R. Hristova and D. A. Horsley, "Scanning Magnetoresistance Microscopy for Imaging Magnetically Labeled DNA Microarrays," Magnetics, IEEE Transactions on, vol. 45, pp. 4816-4820 2009.

[10] M. Pannetier, C. Fermon, G. Le Goff, J. Simola and E. Kerr, "Femtotesla Magnetic Field Measurement with Magnetoresistive Sensors," Science, vol. 304, pp. 1648-1650, June 11, 20042004.

[11] D. Robbes, "Highly sensitive magnetometers--a review," Sensors and Actuators A: Physical, vol. 129, pp. 86-93, 2006.

[12] T. Kagami, et al., "A performance study of next generation's TMR heads beyond $200 \mathrm{~Gb} / \mathrm{in}^{2}$," IEEE Transactions on Magnetics, vol. 42, pp. 93-96, 2006.

[13] R. W. Dave, et al., "MgO-Based Tunnel Junction Material for HighSpeed Toggle Magnetic Random Access Memory," IEEE Transactions on Magnetics, vol. 42, pp. 1935-1939, 2006.

[14] X. F. Han, Z. C. Wen and H. X. Wei, "Nanoring magnetic tunnel junction and its application in magnetic random access memory demo devices with spin-polarized current switching (invited)," vol. 103, pp. 07E933-936, 2008

[15] R. C. Chaves, P. P. Freitas, B. Ocker and W. Maass, "MgO based picotesla field sensors," vol. 103, pp. 07E931-933, 2008.

[16] E. R. Nowak, M. B. Weissman and S. S. P. Parkin, "Electrical noise in hysteretic ferromagnet-insulator-ferromagnet tunnel junctions," Applied Physics Letters, vol. 74, pp. 600-602, 1999.

[17] M. E. Welland and R. H. Koch, "Spatial location of electron trapping defects on silicon by scanning tunneling microscopy," Applied Physics Letters vol. 48, pp. 724-726, 1986.

[18] M. B. Weissman, "1/f noise and other slow, nonexponential kinetics in condensed matter," Reviews of Modern Physics, vol. 60, p. 537, 1988.

[19] X. Liu, C. Ren and G. Xiao, "Magnetic tunnel junction field sensors with hard-axis bias field," Journal of Applied Physics, vol. 92, pp. 4722-4725, 2002

[20] P. W. T. Pong and J. W. F. Egelhoff, "Fabrication strategies for magnetic tunnel junctions with magnetoelectronic applications," San Diego, CA, USA, 2007, pp. 66451V-66411.

[21] Y. Lu, et al., "Shape-anisotropy-controlled magnetoresistive response in magnetic tunnel junctions," Applied Physics Letters, vol. 70, pp. 2610-2612, 1997.

[22] M. Tondra, et al., "Picotesla field sensor design using spin-dependent tunneling devices," Journal of Applied Physics, vol. 83, pp. 6688-6690, 1998.

[23] A. F. M. Nor, et al., "Low-frequency noise in $\mathrm{MgO}$ magnetic tunnel junctions," Journal of Applied Physics, vol. 99, pp. 08T306-303, 2006. 